\title{
Psychosurgery for stuttering
}

This article was published in the following Dove Press journal:

Neuropsychiatric Disease and Treatment

2 April 2015

Number of times this article has been viewed

\section{Edgar Durand' Alexander G Weil ${ }^{2}$ \\ Marc Lévêque ${ }^{1,3}$}

'Espace éthique de l'Assistance publique-Hôpitaux de Paris et Département de recherche en éthique, South Paris University, Paris, France; ${ }^{2}$ Pediatric Neurosurgical Department, Sainte-Justine Hospital, Montreal, QC, Canada; ${ }^{3}$ Service de Neurochirurgie, Hôpital de la Pitié-Salpêtrière, Paris, France
Correspondence: Marc Lévêque Neurosurgical Department, PitiéSalpêtrière Hospital, 47-83 Boulevard de l'Hôpital, 75013 Paris, France Email marclevequemd@gmail.com

\section{Dear editor}

We read with interest the article entitled "Anterior capsulotomy improves persistent developmental stuttering with a psychiatric disorder: a case report and literature review" published in Neuropsychiatric Disease and Treatment. ${ }^{1}$ The authors report on a 28-year-old man with persistent developmental stuttering who was treated by bilateral anterior capsulotomy in the People's Republic of China. To our knowledge, this is a new and previously unreported application of this technique for this indication. Accordingly, as the authors highlight, "the evidence for surgical treatment of persistent developmental stuttering and associated psychiatric disorders is limited", placing their approach within the clinical research forum. In this experimental context, this case report brings forward several important reflections on patient evaluation, technique utilized, and postoperative follow-up.

The authors fail to indicate the patient's maternal language. Given that the team is Chinese, we can assume that it was Mandarin; however, the most widely used variety of Chinese spoken in Sichuan is Sichuanese, which is the lingua franca in Sichuan, Chongqing, and part of Tibet. Either way, for the neuropsychological evaluation scales, it is surprising that the authors refer to articles that have validated these scales in English but not in Chinese. It would have been important for the authors to evaluate the patient's personality before and after the intervention.

Concerning the surgical technique, it is unfortunate that no computed tomography scan was performed to correct for the inherent deformations associated with magnetic resonance imaging (MRI), especially when it is a high-field coregistered 3T MRI. The authors mention that a "test stimulation generated by the Elekta neurostimulator at both high frequency $(130 \mathrm{~Hz})$ and low frequency $(5 \mathrm{~Hz})$ was then carried out to verify the target of the electrode", but they do not indicate the clinical signs that were sought or obtained during stimulation. It is unfortunate that we have no information on the quality of verbal fluency during high and low frequency stimulation. It would have been pertinent for the authors to include the voltage utilized during stimulation. We are not informed whether or not a speech therapist was present perioperatively during this evaluation.

Furthermore, one must question whether a lesional technique was appropriate in this case. The authors' motivation for selecting a bilateral anterior capsulotomy over medical therapy or deep brain stimulation (DBS) was because "both the patient and his family opted for capsulotomy for financial reasons". It would be interesting to know the reasons justifying the bias of burdening the patient with the cost of this supposed biomedical research protocol. Also, when the research is unpublished and founded on weak scientific evidence, a prudent approach is warranted so as to offer the technique associated with minimum risk and maximal benefits. In a research 
context where, like the authors remind us, "the evidence for surgical treatment of persistent developmental stuttering and associated psychiatric disorders is limited", a DBS technique seems justified given that its effects are, contrary to lesional techniques, both reversible and adjustable. From a scientific standpoint, this technique provides the advantage of completing double-blind evaluations and excludes any potential placebo effect contributing to observed improvements. DBS would also have allowed us to verify with which plot and parameters the therapeutic effect was obtained, so as to better understand the underlying pathophysiological mechanisms. Finally, DBS would have permitted electrical recording for several days before connecting the neurostimulator.

The progress made in functional neurosurgery and the emergence of DBS offer an important hope for cure in patients suffering from medically refractory psychiatric illness. We must remain very vigilant regarding the methodology of these studies so that psychosurgery does not, once again, become a matter of controversy in such a way that it compromises its development.

\section{Disclosure}

The authors report no conflicts of interest in this communication.

\section{Reference}

1. Zhang S, Li P, Zhang Z, Wang W. Anterior capsulotomy improves persistent developmental stuttering with a psychiatric disorder: a case report and literature review. Neuropsychiatr Dis Treat. 2014;10: $553-538$. 


\section{Authors' reply}

Shizhen Zhang

Peng Li

Xinjie Zhang

Wei Wang

Department of Neurosurgery, West China Hospital, Sichuan University, Chengdu, People's Republic of China

Correspondence: Wei Wang

Department of Neurosurgery, West China Hospital,

Sichuan University, 37 Guoxue Alley, Chengdu 61004I,

Sichuan Province, People's Republic of China

Emailwcnsww@I63.com

\section{Dear editor}

Thank you for your email and the letter entitled "Psychosurgery for stuttering". Here, I would like to give detailed explanations to the questions raised by Durand et al.

Stuttering severity instrument-3 (SSI-3) includes: frequency, duration, and physical concomitants. The stuttering evaluation scale adopted in our hospital is a comprehensive one which is modified on the basis of SSI-3. The scale includes frequency, duration, physical concomitants and speech naturalness. Although, we cannot find a reference for this modified scale, it has been used for many years in our hospital. Thus, we referred to SSI-3 and speech naturalness scales because our modified scale is based on these two scales, which is simple and convenient for international communication and understanding.

We used shimming before magnetic resonance image (MRI) scan to correct the inherent deformations. A 3T MRI scan was just used to achieve better images and was applied before surgery. A 1.5T MRI and Surgi-plan work station were mainly used to calculate coordinates of the targets and angles of the electrode penetration, which is a routine and widely used neuroimaging method. ${ }^{1}$ Internal capsule was associated with sensation and movement. High frequency stimulation $(130 \mathrm{~Hz})$ and low frequency stimulation $(5 \mathrm{~Hz})$ were carried out separately to find out if there were abnormal sensation (such as numbness) and abnormal movement (such as convulsion). If the patient did not have the abnormal symptoms described, we could verify the target of the electrode. The patient in the case report did not have any abnormal symptoms and it would be better if we described more details of the case report. We used high temperature produced by radiofrequency heating (thermocapsulotomy), but not voltage during stimulation. A speech therapist was present perioperatively during this evaluation, as described in the case report, "Neuropsychological and PDS evaluations were done before surgery and at 6-month follow-up by the same psychiatrist and speech therapist".

We admit deep brain stimulation (DBS) is reversible and adjustable, which is a good option for the patient. Capsulotomy is a technique proven to be safe and effective and is widely reported. ${ }^{1}$ The patient accepted neurosurgery (capsulotomy or DBS), because he would like to "get rid of medicine". As DBS for psychiatric disorders is not covered by the health insurance in the People's Republic of China and the price of DBS is far beyond what the patient and his family could afford, the patient chose capsulotomy. The cost of DBS is paid by health insurance in western countries. It is difficult to discuss the best option for the patient in different cultures and real clinical settings.

Finally, it is emphasized that the alleviation of stuttering was an unexpected outcome, which was the reason we reported the case. The original intention of capsulotomy was to relieve the psychiatric symptoms.

\section{Disclosure}

The authors report no conflicts of interest in this communication.

\section{References}

1. Rück C, Karlsson A, Steele JD, et al. Capsulotomy for obsessivecompulsive disorder: long-term follow-up of 25 patients. Arch Gen Psychiatry. 2008;65(8):914-921.

2. Zhang S, Li P, Zhang Z, Wang W. Anterior capsulotomy improves persistent developmental stuttering with a psychiatric disorder: a case report and literature review. Neuropsychiatr Dis Treat. 2014;10:553-558.

\section{Publish your work in this journal}

Neuropsychiatric Disease and Treatment is an international, peerreviewed journal of clinical therapeutics and pharmacology focusing on concise rapid reporting of clinical or pre-clinical studies on a range of neuropsychiatric and neurological disorders. This journal is indexed on PubMed Central, the 'PsycINFO' database and CAS,

\section{Dovepress}

and is the official journal of The International Neuropsychiatric Association (INA). The manuscript management system is completely online and includes a very quick and fair peer-review system, which is all easy to use. Visit http://www.dovepress.com/testimonials.php to read real quotes from published authors. 